\title{
The Influence of Narrative Education on the Humanistic Caring Quality of Undergraduate Nursing Students
}

\author{
Chang Xuejiao, Wu Jinping, An Xiangli, Zhang Xiuwei* \\ Huzhou University \& First Affiliated Hospital of Huzhou University, Department of Medicine, Huzhou University, Huzhou, China
}

Email address:

1459039166@qq.com (Zhang Xiuwei)

${ }^{*}$ Corresponding author

To cite this article:

Chang Xuejiao, Wu Jinping, An Xiangli, Zhang Xiuwei. The Influence of Narrative Education on the Humanistic Caring Quality of Undergraduate Nursing Students. American Journal of Nursing Science. Vol. 7, No. 5, 2018, pp. 165-168. doi: 10.11648/j.ajns.20180705.11

Received: June 25, 2018; Accepted: August 6, 2018; Published: August 30, 2018

\begin{abstract}
Objective: To explore the effect of narrative education on the quality of humanistic caring of undergraduate nursing students. Methods The Class 2015 and 2016 nursing undergraduates in our college were divided into two groups according to their grades. For the Class 2016 undergraduate nursing students, we used the stories of 73 Chinese Nightingale Prize winner as narrative education materials in the course of humanistic culture. According to the stories, the students were asked to write a speech as an assignment and participate in the speech contests organised in classes and in the school. The humanistic care quality scale was used to investigate the students' humanistic caring quality before and after intervention, and the results were also used to make comparison with the result from students of Class 2015, who did not have humanistic caring courses. Results: The total score ( $\mathrm{t}=3.297, \mathrm{P}=0.001)$, caring concept $(\mathrm{t}=2.784, \mathrm{P}=0.006$ ), caring perception $(\mathrm{t}=3.752, \mathrm{P}<0.001)$, caring ability scores $(\mathrm{t}=2.160, \mathrm{P}=0.032)$ of Class 2016 were higher than grade 2015 nursing students in the level of the same period, and the difference was statistically significant. Conclusion: The method of narrative education based on the stories of Nightingale Prize winner can effectively improve the humanistic caring quality of undergraduate nursing students.
\end{abstract}

Keywords: Narration Education, Humanistic Caring, Teaching Methods, Nursing Students

\section{Introduction}

Humanistic care refers to the nurses' caring for, supporting, and assisting the care receivers to obtain overall physical and mental health and harmony of life. It is also the professional behavior that reflects the caring for life and the humanistic idea [1], and this is the core professional quality of developing quality care services. According to Chen and Liu [2], the humanistic education can help to cultivate high-quality talents with humanistic caring abilities. However, currently in China, the main receiver of humanistic education is still teachers, resulting in students' emotion, to some extent, ignored [3-4]. Therefore, the nursing students lack professional self-identity, as well as the understanding and enthusiasm for humanistic care. According to Diekelmann [7], narrative pedagogy is a teaching method that aims to educate the students by explaining, analysing and reconstructing the life experience of students, teachers and clinical nurses. Andrews et al. [8] view it as a method in which educators and students work together in challenge-based learning, practice reflection and experience interpretation. And Ironside believes that narrative pedagogy is a phenomenological approach to teaching and learning based on research [9]. In humanistic education, the shift of focus from course content to the learner's emotional experience can help to develop nursing students' caring ability and professional self-identity [10-11]. Based on such theories, the narrative education in our study is an education method which involves the teachers and students to make a joint effort in organising, analysing and reconstructing the work experience of the Nightingale Prize winners, to reflect on, interpret and communicate such experience and finally to construct the ideal professional image and values.

\section{Study Subjects}

92 first year full-time undergraduate students in Class 2016 
in Huzhou University were recruited as our study subjects. Among them, 88 students were female and 4 were male; their age ranged from 18 to $20(19.97 \pm 0.60)$ years old.

\section{Methods}

\subsection{Teaching Methods}

We have chosen The Humanistic Quality Cultivation for Nurses edited by Li Huiling and Zhang Xiuwei as the textbook in the course of humanistic culture. There were 32 credit hours, including 18 hours for the theory, 10 hours for practice and 4 hours for assessments. The content of theory courses mainly included humanistic culture and values, care and responsibility, love and respect, language and behavior, observation and analysis ability and physiological care, empathy coordination ability and psychological care, artistic accomplishment and humanistic environment, etc. The content of the practical lessons included the observation and experience of patients' needs, behavior training, language training, excellent nurses' experience of patient's physical and mental care, and the design show of "Nursing Humanistic Environment". For assessments, tests were given to evaluate the students' level of comprehension and a speech event on the stories of Nightingale Prize winner was organised to assess the students' quality of humanistic care.

Narrative pedagogy was also added to the course on the bases of the humanistic content. In each class, the teacher played a 5 to 10 -minute narrative clip, including domestic and foreign caring stories and videos. And the students should collect and organise the information, write a speech, participate in the preliminary speech test within the classes and the finals in the school. The content that students needed to collect and organise consisted of three parts - the awards ceremony (the time, place and awards), the prize winners' personal introduction and their stories. In this process, students were required to consult high-quality documents to ensure the authenticity of the stories. The information organised and the speech written were submitted for assessment with such criteria: text with pictures provided $(20 \%)$, well formatted $(20 \%)$, realistic and touching $(30 \%)$ and profound understanding (30\%).

The preliminary speech contest took 4 practical lessons, with 30 students being the contestants ( 24 were voluntary participants and 6 were randomly selected). Each student was given 5 minutes to deliver the speech and the speeches were evaluated by their teachers and peers who were not contestants under this standard: novelty of the theme (20\%), informativeness (20\%), profoundness of understanding (40\%) and vividness of language (20\%). Students got high scores in the preliminary contests could participate in the finals and each was given 6 minutes to deliver the speech. In the finals, the judges were their teachers of the course, student representatives and teachers from the department. The comments were given orally by the leaders of the department and the school and the winners of the $1^{\text {st }}, 2^{\text {nd }}, 3^{\text {rd }}$ prize were selected according the following criteria: appropriately dressed (5\%), natural facial expression (5\%), accuracy $(5 \%)$, fluency $(10 \%)$, proper volume and rhythm $(10 \%)$, well structured $(10 \%)$, conciseness $(20 \%)$ and highlighting of the theme $(20 \%)$.

\subsection{Effect Evaluation Methods}

We used humanistic care quality scale to assess the students before and after the intervention and we also compared the results with the scores we got from the students of Class 2015 who did not have narrative education. 91 students of Class 2015 were selected in our study. Among them, 3 were male and 88 were female; their age was 19-21 (20.13 士 0.60). For these students, the number of credit hours and the textbook were same as their Class 2016 counterparts, and they also had theory classes, clinical experience, nursing home service experience and role play practice. And these students were encouraged to read the notes written by Florence Nightingale, as well as the caring theories. The assessments included theory test that evaluated the students' mastery of theoretical knowledge and role play performance which helped assess the students' humanistic quality.

The assessment tool we have chosen was the humanistic care quality scale (nursing student edition) edited by Liu Yuxiao [12]. The scale includes a total of 29 items in four dimensions: idea of care, knowledge of care, caring ability and caring perception. Using Likert-type 5-level method, the options of each question are from disagreement ( 1 score) to agreement (5 scores). The higher total score of this scale indicates the better humanistic care quality the students have. The Cronbach's acoefficient of the whole scale is 0.913 and the coefficient of all the dimensions range from 0.714-0.840.

The scales were distributed by the researchers in classes for the students to complete independently and anonymously after informed consent. All the students we recruited in the study had completed the scale before and after the intervention, and the valid response rate was $100 \%$.

\subsection{Statistical Method}

The statistical software SPSS21.0 was used to analyse the data. The measurement data such as students' care quality scores were expressed as $\bar{X} \pm \mathrm{s}$ and the data from two groups were compared using independent sample t-test.

\section{Results}

Before the students had humanistic culture course, there was no significant difference in the total score and each dimension of humanistic care quality between students from Class 2016 and Class 2015 ( $\mathrm{P}>0.05$ ) (Table 1). After having the course, students from Class 2016 showed significantly higher total scores and higher scores in the dimensions of idea of care, caring ability and caring perception than their counterparts in Class $2015(\mathrm{P}<0.05)$, and had no significant difference from Class 2015 in the dimension of knowledge of caring $(\mathrm{P}>0.05)$ (Table 2). 
Table 1. The comparison of students'scores of caring quality between Class 2016 and Class 2015 before intervention (score, $\bar{X} \pm S$ ).

\begin{tabular}{lllll}
\hline Item & Class 2016 $(\mathbf{n}=\mathbf{9 2})$ & Class 2015 $(\mathbf{n}=\mathbf{9 1})$ & $\mathbf{t}$ & P \\
\hline Idea of care & $29.08 \pm 4.38$ & $29.18 \pm 4.70$ & 0.148 & 0.882 \\
Knowledge of care & $30.43 \pm 1.79$ & $30.21 \pm 2.35$ & 0.203 & 0.840 \\
Caring ability & $30.13 \pm 4.50$ & $30.15 \pm 5.11$ & 0.031 & 0.975 \\
Caring perception & $32.67 \pm 3.88$ & $32.55 \pm 3.71$ & 0.222 & 0.825 \\
Total score & $122.32 \pm 14.54$ & $122.09 \pm 18.37$ & 0.093 & 0.926 \\
\hline
\end{tabular}

Table 2. The comparison of students'scores of caring quality between Class 2016 and Class 2015 after intervention (score, $\bar{X} \pm S$ ).

\begin{tabular}{lllll}
\hline Item & Class 2016 $(\mathbf{n}=\mathbf{9 2})$ & Class 2015 $(\mathbf{n}=\mathbf{9 1})$ & $\mathbf{t}$ & $\mathbf{P}$ \\
\hline Idea of care & $31.95 \pm 2.83$ & $30.99 \pm 1.67$ & 2.784 & 0.006 \\
Knowledge of care & $30.79 \pm 2.06$ & $30.61 \pm 2.42$ & 0.536 & 0.593 \\
Caring ability & $31.60 \pm 5.50$ & $30.51 \pm 1.47$ & 2.160 & 0.032 \\
Caring perception & $33.76 \pm 1.33$ & $33.03 \pm 1.29$ & 3.752 & $<0.001$ \\
Total score & $128.10 \pm 8.01$ & $125.14 \pm 2.99$ & 3.297 & 0.001 \\
\hline
\end{tabular}

\section{Discussion}

\subsection{The Method of Narrative Pedagogy Is Helpful to the Improvement of Students' Humanistic Caring Quality}

After the course, students from Class 2016 had significantly higher total scores and scores in the dimensions of idea of care, caring ability and caring perception than their counterparts in Class $2015(\mathrm{P}<0.05)$, indicating that narrative education can help to improve students' humanistic care quality.

Humanistic care has three aspects: 1) the nurses' sympathy, respect, protection and care based on the perception of the value of life and nobleness of humanity, 2) the sense of responsibility to meet the needs of care receivers, and 3) the rational knowledge of humanitarian belief and value [13]. Humanistic care perception is the perceptual knowledge of the state of feeling, emotional experience and level of satisfaction that nurses, as a dual role of caring and the cared-for, obtain [13]. It shows that the narrative education based on the stories of Nightingale Prize winners plays a crucial role in improving students' humanistic care quality, in both rational and emotional ways. This is consistent with the conclusion of similar studies, and is also reflected in the students' speech:

"Following the example of the Nightingale Prize winner, Pan Meier, I will treat patients with a sincere heart. We will become excellent nurses like her, as she said, 'there is only remote mountains, no remote life."

"Florence Nightingale, it is you that led me to find my belief, and I know that my course of life is to care for people wholeheartedly."

Humanistic care ability is the relevant skills nurses need to implement patient care activities [13]. The result of this study is consistent with some other studies [11, 16-17] that narrative education can improve students' empathy experience, moral support, emotional contact, observation of symptoms and caring ability to the cared-for. In addition to this, the caring ability in our study also includes professional insights, situation analysis, interpersonal coordination and problem-solving skills. As some students said:

"I will follow the example of Director Zou Ruifang: Even if the patients don't speak, I should know what they need with merely a look or a move."

"In the caring for the special patients, I will respect the patients' cultural backgrounds, social roles, personality traits and other factors like our predecessor Liu Shuyuan. Many nurses thought it unreasonable when a mother asked the nurse to read nursery rhymes to her child with Down's syndrome, but Ms. Liu, unexpectedly, asked the nurse on duty to read rhymes in a lively tone, which brought the mother much comfort."

There was no significant difference in the dimension of caring knowledge between the two groups of students $(\mathrm{P}>0.05)$, which is not consistent with the result in He's study [18]. The reason to this inconsistency might be that in He's study, they began to provide narrative education only after giving the course of basic nursing theory in students' second year. As Guo [19] has said, "Adding narrative education to the professional courses can promote students' understanding of caring knowledge." However, in our study, we have provided narrative education to freshmen who have not had any professional courses, leading to less desirable result in the increasing of knowledge.

\subsection{Summary of Experience}

The stories from clinical nursing practice is closer to the reality. Because of the its guiding and inspiring effects, it can bring the students closer to the stories, make it easier for them to engage in the scenarios, help them to find their examples and find the directions of their efforts [20]. By using the stories of Nightingale Prize winner as materials of narrative education, students can experience the emotion changes similar to that in real situations while organising relevant information, and thus able to deliver their humanistic feelings and idea of care and concern in their speeches. As Tang [21] said, "Narrative is a process of explore the moral educational resources from within an individual. When the narrator combines his/her own experience with the stories of their role models, it can enhance the narrator's understanding of human nature and his/her internalization of humanistic caring ideas." Through the teachers and students' joint effort in working together in understanding the stories, they were able to find the unique value of nursing profession and thus enhance their professional self-identity and sense of mission [22-23]. 
However, some students thought that collecting data is only a homework and did it passively. To cope with students' lack of interest and carelessness of reading, we had taken two solutions: in addition to the video played in classes, we invited two Nightingale Prize winners to give speeches in our school and allowed students to take photos with them, which provided objective basis and motivation for their information collection; for shy and introvert students, we provided speech videos and speech demonstrations by their teachers to encourage and motivate them. Awards were provided too.

\section{Conclusion}

The narrative education based on the stories of Nightingale Prize winner contributes to the development of students' humanistic care quality. Our study still have some limitations, like the short time ( $5 \mathrm{~min}$ ) for each student in the preliminary contest, which hardly allows students to fully express themselves. We should apply narrative education in more courses and the clinical training to help students form stable humanistic quality.

\section{References}

[1] Zhang Xiuwei, Jiang Anli. Current situation and analysis of the concept of nursing humanistic care [J]. Chinese Journal of nursing, 2008, 43 (6): 540-543.

[2] Chen Rui, Liu Yilan. Research status of nursing humanistic care curriculum [J]. Chinese Journal of nursing, 2014, 49 (10): 1249-1253.

[3] Mu Ronghong, Li Qiong, Li Nana. Reflections on the reform of humanistic quality education in higher nursing education [J]. Chinese nursing education, 2014, 11 (2): 149-151.

[4] Qiao Xue, Hao Yufang. Strategy of humanistic care education in nursing teaching in Chinese universities [J]. Chinese nursing education, 2015, 12 (8): 639-641.

[5] Zhou Ying, Hao Yanping. Analysis and Countermeasures of humanistic education in higher nursing education in China [J]. Journal of nursing management, 2010, 10 (5): 347-349.

[6] Zhang Jinhua, Gao Min, Li Wei, et al. Survey of humanistic caring ability of undergraduate nursing students $[\mathrm{J}]$. Chinese nursing education, 2012, 9 (7): 328-330.

[7] Diekelmann N. Narrative pedagogy; Hcideggarian hermeneutical analyses of lived experiences of students, teachers and clinicians [J]. Adv Nurs Sci, 2001, 23 (3):53-71.

[8] Andrews CA, Ironside PM, Nosek C, et al. Enacting narrative pedagogy: the lived experiences of students and teachers [J]. Nurs Health Care Perspect. 2001, 22 (5):252-259.
[9] Ironside PM. Enabling narrative pedagogy: inviting, waiting, and letting be [J]. Nurs Educ Perspect, 2014, 35 (4):212-218.

[10] Guo Yujie, Jiang Anli. Application of narrative education in nursing education $[\mathrm{J}]$. Journal of nursing studies, 2011, 26 (1): 25-26.

[11] Li Xiangyan, Zhao Tiyu, Jiang Li, et al. Application of narrative education in clinical teaching of nursing undergraduate interns in operation room $[\mathrm{J}]$. nursing journal, 2017, 32 (2): 63-66.

[12] Liu Yuxiao, Jiang Anli. Development of nurse humanistic care quality assessment scale [J]. PLA Journal of nursing, 2012, 29 (16): 19-21, 25.

[13] Zhang Xiuwei. Construction of theoretical model of nursing humanistic care quality structure and educational strategy [D]. Shanghai: Second Military Medical University, 2009.

[14] Zhao Hong, Yao Pingbo, Tang antelope, et al. Application of narrative education in psychiatric nursing teaching [J]. general nursing, 2015, 13 (29): 2976-2977.

[15] Dong Yongze, Yang Qingmin, Qiu Zhichao. Application of narrative nursing teaching in Nursing Humanities training course [J]. Chinese nursing education, 2016, 13 (9): 675-679.

[16] Yuan Jinrong, Liu Yilan, Yang Sai. Application of narrative education in cultivating humanistic care ability of nursing students [J]. nursing journal, 2016, 31 (1): 14-16.

[17] Cai Qin, Fang Jun, Zong Wei, et al. Using the narrative teaching method to carry out humanistic care education for psychiatric nursing students. [J]. nursing study journal, 2016, 30 (1): 61-63.

[18] He Liping, Zhou Yun. Application of narrative education in cultivating the humanistic care quality of nursing undergraduates $[\mathrm{J}]$. Chinese medicine guide, 2015, 12 (9): 157-159.

[19] Guo Yujie, Shen Wang Qin, Song Yan, and so on. "Caring narrative" teaching model applied to the teaching of nursing undergraduate "foundation of nursing" [J]. nursing journal, 2014, 21 (4): 4-7.

[20] Gao Chenchen, Jiang Anli. Development and production of narrative nursing materials for humanistic care [J]. Journal of PLA nursing, 2014, 31 (7): 15-18.

[21] Tang Shengwei. The transfer of research paradigms in foreign narratology -- Commenting on the current situation of narratology in China $[\mathrm{J}]$. Journal of Sichuan International Studies University, 2003, 19 (2): 15-19.

[22] Walsh M. Narrative pedagogy and simulation:future directions for nursing education [J]. Nurse Educ Pract, 2011, 11 (3):216-219.

[23] Gao Chenchen, Jiang Anli. Application of narrative education in nursing humanistic care education [J]. PLA Journal of nursing, 2013, 30 (11): 31-33. 